\title{
Breaking Linear Scaling Relationships with Secondary Interactions in Confined Space: A Case Study of Methane Oxidation by Fe/ZSM-5 Zeolite
}

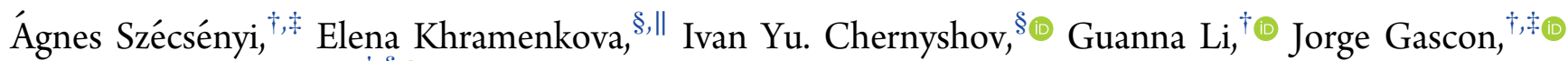
and Evgeny A. Pidko* ${ }^{*}, \S_{\odot}$

${ }^{\dagger}$ Department of Chemical Engineering, Delft University of Technology, Van der Maasweg 9, 2629 HZ Delft, The Netherlands

${ }^{\ddagger}$ King Abdullah University of Science and Technology, KAUST Catalysis Center, Advanced Catalytic Materials, Thuwal 23955, Saudi Arabia

${ }^{\S}$ TheoMAT Group, ChemBio Cluster, ITMO University, Lomonosova Street 9, Saint Petersburg, 191002, Russian Federation

Supporting Information

ABSTRACT: Linear energy scaling laws connect the kinetic and thermodynamic parameters of key elementary steps for heterogeneously catalyzed reactions over defined active sites on open surfaces. Such scaling laws provide a framework for a rapid computational activity screening of families of catalysts, but they also effectively impose a fundamental limit on the theoretically attainable activity. Understanding the limits of applicability of the linear scaling laws is therefore crucial for the development of predictive models in catalysis. In this work, we computationally investigate the role of secondary effects of the active site environment on the reactivity of defined $\mathrm{Fe}$ complexes in ZSM-5 zeolite toward methane oxofunctionalization. The computed $\mathrm{C}-\mathrm{H}$ activation barriers over Fe-sites at different locations inside the zeolite pores generally follow the associated reaction enthalpies and the hydrogen affinities of the active site, reflecting the $\mathrm{O}-\mathrm{H}$ bond strength. Nevertheless, despite the close similarity of the geometries and intrinsic reactivities of the considered active complexes, substantial deviations from these linear scaling relations are apparent from the DFT calculations. We identify three major factors behind these deviations, namely, (1) confinement effects due to the zeolite micropores, (2) coordinative flexibility, and (3) multifunctionality of the active site. The latter two phenomena impact the mechanism of the catalytic reaction by providing a cooperative reaction channel for the substrate activation or by enabling the stabilization of the intrazeolite complex along the reaction path. These computational findings point to the need for the formulation of multidimensional property-activity relationships accounting for both the intrinsic chemistry of the reactive ensembles and secondary effects due to their environmental and dynamic characteristics.

KEYWORDS: methane, methanol, selective oxidation, DFT calculations, reaction mechanism, zeolites

\section{INTRODUCTION}

The development of predictive models to guide and facilitate the discovery and optimization of catalysts for important chemical applications has been a central problem in catalysis research for decades. ${ }^{1,2}$ The identification of representative equilibrium states that could be used as performance descriptors under the nonequilibrium conditions is one of the cornerstones of modern catalysis research. Such descriptors reflect the intrinsic chemistry of the catalytic site and may be related to structural features, ${ }^{3}$ electronic properties, ${ }^{4}$ or thermodynamics. ${ }^{5}$ Impressive progress has been made in the past decade in the development of universal scaling relationships that correlate linearly the barriers of elementary reaction steps with the binding or adsorption energies for key intermediates through Brønsted-Evans-Polanyi (BEP) rela- tionships. ${ }^{6-8}$ Such linear relationships facilitate greatly the in silico search for an optimal catalyst and enable practical applications of machine learning algorithms in computational catalysis ${ }^{9,10}$ by significantly reducing the number of independent parameters that determine catalytic reactivity. Scaling relations provide a theoretical framework for constructing volcano plots from first-principles and therefore put fundamental limits on the maximum achievable activity or selectivity for a particular catalyst class. ${ }^{11,12}$

A representative example of the universal scaling relationship is the linear correlation between the activation barrier for

Received: May 8, 2019

Revised: $\quad$ August 27, 2019

Published: September 4, 2019 
A

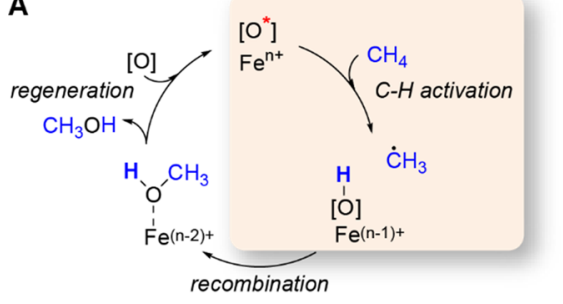

C

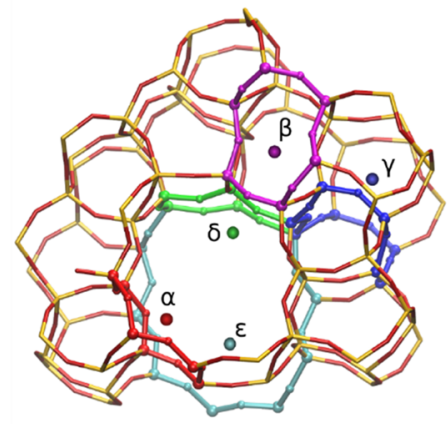

B
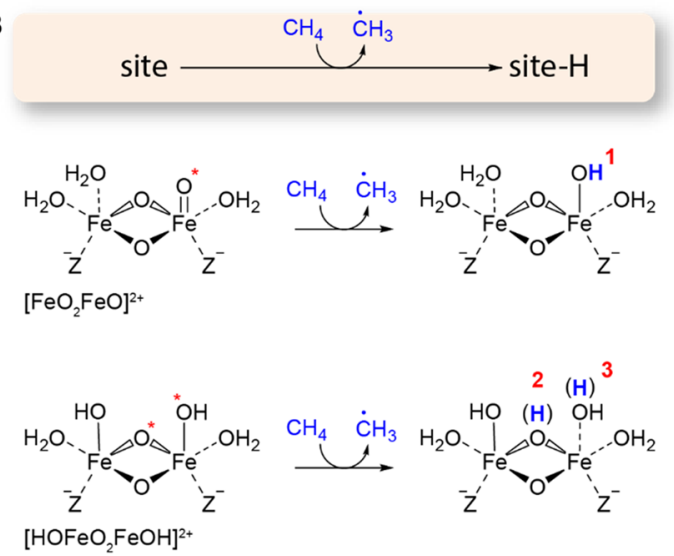

$\mathrm{HOFeOFe}_{\mathrm{H}_{2} \mathrm{O} \cdot \mathrm{O}^{\mathrm{HO}} \mathrm{OH}^{*} \mathrm{O}^{2+}}^{\mathrm{O}^{2+}}$

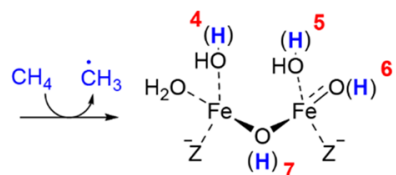

Figure 1. (A) The key steps of the catalytic oxo-functionalization of methanol over an oxygenated iron site representing (B) the three binuclear Fe catalytic sites ("site") featuring reactive oxygen sites (indicated with $*$ ) able to initiate the homolytic $\mathrm{C}-\mathrm{H}$ cleavage in methane resulting in a ${ }^{\bullet} \mathrm{CH}_{3}$ species and a partially reduced complex ("site-H"). The cationic di-Fe complex can be stabilized at (C) different extraframework positions in the pores of ZSM-5 featuring versatile coordination and confinement environment.

homolytic methane $\mathrm{C}-\mathrm{H}$ bond activation and hydrogen affinity. The latter thermodynamic descriptor holds for various solid catalysts, ranging from transition metal surfaces to zeolites and oxides. ${ }^{13}$ This correlation could allow for the largescale computational screening of materials to identify new heterogeneous catalysts capable of efficiently promoting the oxidation of methane to methanol. Such a catalytic process could revolutionize the natural gas industry by providing a practical means for the one-site liquefaction and valorization of small-scale remote sources. ${ }^{14-16}$ A similar correlation between the reactivity of zeolites toward homolytic methane $\mathrm{C}-\mathrm{H}$ bond cleavage and spin-density of the active oxygen site was also proposed for $\mathrm{Cu} / \mathrm{ZSM}-5$ zeolite. ${ }^{17,18}$ Snurr et al. extended the linear scaling relationship to metal-organic framework (MOF) based catalysts and pointed out that the reactivity for methane $\mathrm{C}-\mathrm{H}$ bond activation has a uniform correlation with the formation energy of the active oxygen site in 60 different MOF structures. ${ }^{19}$ Despite the clear generality of these relationships provided by the major impact of the intrinsic chemistry of the active site on the reactivity, substantial deviations from the general trends of up to $70 \mathrm{~kJ} / \mathrm{mol}$ could be seen in the reported general trends. Understanding the origin of these deviations may provide design principles allowing to circumvent the associated fundamental limitations on the catalyst activity. $^{20-23}$

Secondary interactions with the active site environment may influence the shape of the potential energy surface corresponding to the reaction and product states and thus provide a mechanism to break the primary scaling relations. These interactions are particularly important for catalytic reactions in microporous materials such as $\mathrm{MOFs}^{24,25}$ and zeolites. ${ }^{26-28}$ Fe-containing zeolite and MOF systems are among the promising catalysts for the selective $\mathrm{C}-\mathrm{H}$ oxidation and their multinuclear cationic Fe-oxo/hydroxo complexes are often regarded as synthetic mimics of the methane monooxygenaze enzyme. $^{29-31}$ In this study we investigate in detail how variations in the local environment of such binuclear Fe(IV) clusters deposited in the ZSM-5 zeolite matrix affect their reactivity toward homolytic $\mathrm{C}-\mathrm{H}$ activation in methane. A detailed analysis of the factors leading to the deviation from the linear relationships was carried out. As a result, we identify the mechanisms that can be utilized in catalyst design toward enhanced reactivity beyond the fundamental limits of the scaling relations.

\section{COMPUTATIONAL DETAILS}

The spin polarized periodic DFT calculations were carried out with the Vienna $\mathrm{Ab}$ Initio Simulation Package (VASP 5.3.5). ${ }^{32,33} \mathrm{PBE}$ exchange-correlation functional ${ }^{34}$ was used together with the plane wave basis set with a kinetic energy cutoff of $450 \mathrm{eV}$ and the projector augmented wave (PAW) method. ${ }^{35}$ To account for the van der Waals interactions, the semiempirical Grimme's dispersion correction with BeckeJonson damping (DFT-D3(BJ)) method was used. ${ }^{37}$ Our previous study on methane activation via a radical rebound path by a related $\mathrm{Cu}$-zeolite catalyst system support the selection of the current computational methodology. ${ }^{36}$ The calculations at the PBE-D3 and a higher-level multireference CASPT2 level yielded qualitatively similar energetics and electronic structures of key reaction intermediates in those systems. A Gaussian smearing of the population of partial occupancies with a width of $0.05 \mathrm{eV}$ was used during iterative diagonalization of the Kohn-Sham Hamiltonian. Brillouin zone sampling was restricted to the Gamma point. Convergence was assumed to be reached when the force on each atom was below $0.04 \mathrm{eV} \AA^{-1}$. The unit cell lattice parameters were optimized and fixed throughout the calculations $(a=20.1 \AA, b=19.8 \AA, c=13.2 \AA, \alpha=\beta=\gamma$ $=90^{\circ}$ ).

To locate the transition states, the climbing image nudged elastic band method (CI-NEB) ${ }^{38}$ was applied. The maximum energy geometry along the reaction path generated by the NEB method was further optimized using a quasi-Newton algorithm. At this step, only the extra-framework atoms of 


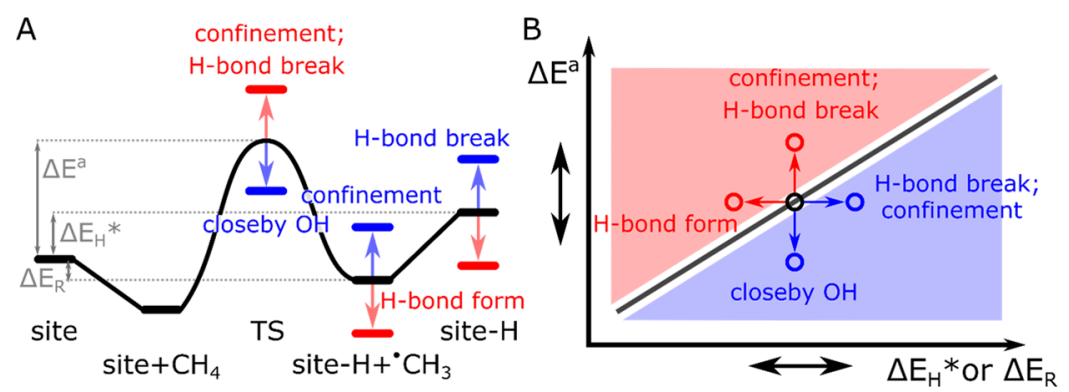

Figure 2. Generalized (A) energy diagram and (B) linear energy relationships $\left(\Delta E^{\mathrm{a}}-\Delta E_{\mathrm{R}}\right.$ and $\left.\Delta E^{\mathrm{a}}-\Delta E_{\mathrm{H}}{ }^{*}\right)$ for the rebound mechanism of $\mathrm{CH}_{4}$ with schematic indication of the influence of the secondary effects on the energetics of the individual states and the associated deviations from the fundamental scaling laws. Negative deviations due to TS destabilization or FS stabilization are shown in red, while the blue color is used to indicate effects resulting in lower TS energies and relatively higher energies of FS giving rise to the apparently higher reactivity of the active site than predicted by the idealized energy scaling relationship.

the reactive center and methane molecule were fully relaxed, while the remaining atoms of the zeolite lattice were fixed. Vibrational frequencies were calculated using the finite difference method (0.02 $\AA$ atomic displacements) as implemented in VASP. Transition state showed a single imaginary frequency corresponding to the reaction path.

Intrazeolite sites were simulated using full periodic unit cells of the MFI zeolite, whereas the open sites at the outer surface were represented by periodic slab models of the (001) MFI surface. The unit cell of the zeolite was truncated in the $\mathrm{z}$ direction along the $\mathrm{ab}$ surface. All terminal dangling $\mathrm{O}$ atoms were saturated with $\mathrm{H}$ atoms. A $20 \AA$ vacuum layer was placed between the slabs to ensure a negligible interaction between the surface species and the next periodic image. Gamma point was used to sample the Brillouin zone.

All data analysis and discussion in this work have been done based on the relative electronic energies. The justification of this choice is elaborated in the Supporting Information (SI), where a series of test calculations for Gibbs free energy is presented. The inclusion of the entropic corrections did not significantly change the relative energy trends. All calculations were carried out at the high spin (HS) potential energy surface. The HS state was always calculated based on the oxidation state of the $\mathrm{Fe}$ centers and the number of radical species present in the system. For example an $\mathrm{Fe}(\mathrm{III})$ dimer has $53 \mathrm{~d}$ electrons on each Fe center, therefore the high spin state is $S=$ 10/2. Each Fe site in the oxidized formally Fe(IV) dimer bears 43 d electrons giving $S=8 / 2$ as the high spin state. Although the most stable spin state in such systems is often the antiferromagnetically coupled high spin configuration (AHS), it has been demonstrated earlier that the reactivity of Obridged Fe complexes is similar in the HS and AHS states. ${ }^{39}$ An extensive justification for such a model choice can be found in ref. ${ }^{40}$

Impact of several secondary interactions on the $\Delta E^{\mathrm{a}}$ deviation were tested using multiple regression. For the purpose six predefined confinement and H-bonding descriptors which had a physical explanation for their effect on energy deviation were tested. We did not test other descriptors in order to avoid choice-supportive bias, as our goal was not to find the best descriptor for 24 data points, but to show that effects of secondary interactions on activation energy can be modeled even with some simple geometry-based descriptors. Adjusted R-squared value was used as a model selection criterion.

\section{RESULTS AND DISCUSSION}

The rebound mechanism of methane oxidation by a $\mathrm{Fe}-\mathrm{O}$ moiety is depicted in Figure 1A. In this work we specifically focus on the first step of the catalytic cycle, the homolytic C$\mathrm{H}$ bond activation over representative binuclear $\mathrm{Fe}$ sites (Figure 1B). The reactive sites in Fe/ZSM-5 take form of extraframework binuclear $\mathrm{Fe}(\mathrm{IV}) \mu$-oxo-bridged complexes with an octahedral coordination environment of the iron centers completed by the lattice oxygen, extraframework terminal oxo and hydroxo- ligands and water molecules. The formation of such complexes upon oxidation of the stable $\left[\left(\mathrm{H}_{2} \mathrm{O}\right)_{2}-\mathrm{Fe}(\mathrm{III})-(\mu-\mathrm{O})_{2}-\mathrm{Fe}(\mathrm{III})-\left(\mathrm{H}_{2} \mathrm{O}\right)_{2}\right]^{2+}$ precursors (Figure $\mathrm{S1}$ ) with $\mathrm{H}_{2} \mathrm{O}_{2}$ has been discussed in detail in our previous work..$^{39,41}$ Note that each of the clusters presents a number of reactive oxygen centers suitable to initiate the homolytic $\mathrm{C}-\mathrm{H}$ cleavage. The reactive oxygens considered explicitly in this study are denoted with an asterisk $(*)$ and Arabic numerals in Figure 1B. The binuclear clusters were accommodated inside the ZSM-5 pores at different extraframework positions (Figures $1 \mathrm{C}$ and S2) with varied confinement and coordination properties. The $\alpha$ and $\delta$ sites are the 6 member-ring (MR) units located along the main channel of ZSM-5, and the $\varepsilon$ site represents a cation position across it. The $\beta$ and $\gamma$ sites are, respectively, the $6 \mathrm{MR}$ and $8 \mathrm{MR}$ rings on the wall of the sinusoidal channel. Besides these intrazeolite cation sites, open site models (site o) were included in our analysis to simulate a less confined environment of the binuclear Fe sites located at the outer surface of the zeolite. The coordination properties and topology of this surface cation site is equivalent to the $\gamma$ site. The combination of the Arabic numeral indicating the proton-accepting site of the diFe cluster, and the Greek symbol for the position in the zeolite are used to differentiate the reactive models studied here. For some sites, we identified several alternative reaction channels with different energetics starting from different adsorption configurations of methane. These reaction channels are distinguished by an $*$ symbol(s) in the notation of the active site. The local optimized geometries of all sites are shown in Figure S2.

Despite binuclear Fe sites can initiate different mechanisms of $\mathrm{C}-\mathrm{H}$ activation, in this study we specifically focused on the homolytic $\mathrm{C}-\mathrm{H}$ cleavage/radical rebound mechanism widely accepted as the dominant path in methane to methanol oxidation by zeolite-based and generally site-isolated heterogeneous catalysts. ${ }^{42,43}$ A simplified reaction energy profile, state and energy definitions used in this study are schematically 
summarized in Figure 2A. The reference state is the noninteracting active site in the periodic zeolite model and a free methane molecule in the gas-phase (site). The reactions start from the state site $+\mathrm{CH}_{4}$ representing an adsorption complex of methane at the zeolite active site, from which the $\mathrm{C}-\mathrm{H}$ bond cleavage via a homolytic transition state (TS) can take place to form a $1 \mathrm{e}^{-}$reduced di-Fe complex and a ${ }^{\bullet} \mathrm{CH}_{3}$ species ( site-H $\left.+{ }^{\bullet} \mathrm{CH}_{3}\right)$. The next step is the recombination of the partially reduced site and the radical to form methanol or a grafted methoxy species. ${ }^{17}$ This recombination step is chemically independent of the initial $\mathrm{C}-\mathrm{H}$ activation and therefore is not considered in this study devoted to the analysis of the secondary effects on the energy scaling relations for homolytic $\mathrm{C}-\mathrm{H}$ activation. The thermodynamic cycle however can be established between the product of homolytic $\mathrm{C}-\mathrm{H}$ cleavage (site- $\mathrm{H}+{ }^{\bullet} \mathrm{CH}_{3}$ ) and the $\mathrm{H}$-affinity of the active site, which is formed upon the desorption of a ${ }^{\circ} \mathrm{CH}_{3}$ radical. Such a configuration can be formed when radical recombination takes place at a distant site leaving behind the reduced Fe complex (site-H). Below we refer to both site- $\mathrm{H}+{ }^{\bullet} \mathrm{CH}_{3}$ and site- $\mathrm{H}$ as the final states (FSs) of the methane activation reaction.

Following the above definition of the main states, we define the reaction energy $\Delta E_{\mathrm{R}}$, apparent activation energy $\left(\Delta E^{\mathrm{a}}\right)$, and $\mathrm{H}$-affinity $\left(\Delta E_{\mathrm{H}}\right)$ as follows:

$$
\begin{aligned}
& \Delta E_{\mathrm{R}}=E\left(\text { site-H }+{ }^{\bullet} \mathrm{CH}_{3}\right)-E(\text { site })-E\left(\mathrm{CH}_{4}\right) \\
& \Delta E^{\mathrm{a}}=E(\mathrm{TS})-E(\text { site })-E\left(\mathrm{CH}_{4}\right) \\
& \Delta E_{\mathrm{H}}^{*}=E(\text { site-H })+E\left({ }^{\bullet} \mathrm{CH}_{3}\right)-E(\text { site })-E\left(\mathrm{CH}_{4}\right) \\
& \Delta E_{\mathrm{H}}=E(\text { site-H })-E(\text { site })-\frac{1}{2} E\left(\mathrm{H}_{2} \mathrm{O}\right)+\frac{1}{4} E\left(\mathrm{O}_{2}\right)
\end{aligned}
$$

where $E\left(\mathrm{H}_{2} \mathrm{O}\right), E\left(\mathrm{O}_{2}\right), E\left(\mathrm{CH}_{4}\right)$, and $E\left({ }^{\bullet} \mathrm{CH}_{3}\right)$ are the DFTcomputed energies of the respective molecules in the gas phase. Although the $\mathrm{H}$-affinity $\Delta E_{\mathrm{H}} *$ given by (3) can directly be related to the thermodynamic cycles of the investigated process, as shown in Figure 1A, in the discussion below a generalized $\mathrm{H}$-affinity $\Delta E_{\mathrm{H}}$ defined in ref 13 and given with eq 4 will be used as a descriptor to facilitate the comparison between the current results and those reported in the literature. $\Delta E_{\mathrm{H}}$ was originally introduced as a universal descriptor for the reactivity of all types of catalysts toward homolytic $\mathrm{C}-\mathrm{H}$ bond cleavage. ${ }^{13}$ It is computed as the reaction energy for the reduction of the protonation and one-electron reduction of a given active site accompanied by gas-phase $\mathrm{O}_{2}$ and $\mathrm{H}_{2} \mathrm{O}$ used as the reference reactants. The two parameters differ by a constant corresponding to the energy difference in the molecular reference states. The slope of the fundamental $\Delta E^{\mathrm{a}}-\Delta E_{\mathrm{H}}$ and $\Delta E^{\mathrm{a}}-\Delta E_{\mathrm{H}} *$ relationships should therefore be the same and any deviation from it is not influenced by the choice of the equation.

Figure $3 \mathrm{~A}$ and $\mathrm{B}$ show the relationships between the computed values of $\Delta E^{\mathrm{a}}$ and the reaction energies $\left(\Delta E_{\mathrm{R}}\right)$ and hydrogen affinities $\left(\Delta E_{\mathrm{H}}\right)$, respectively, with the corresponding linear fits. The $\Delta E_{\mathrm{R}}$ descriptor accounts for (de)stabilization of both ${ }^{\circ} \mathrm{CH}_{3}$ and $\mathrm{H}-[\mathrm{O}]$ reactive species forming in the TS resulting in a better linearity of the respective $\Delta E^{\mathrm{a}}-\Delta E_{\mathrm{R}}$ correlation $\left(R^{2}=0.754\right.$, Figure $\left.3 \mathrm{~A}\right)$ compared to the more general $\Delta E^{\mathrm{a}}-\Delta E_{\mathrm{H}}$ relationship $\left(R^{2}=0.422\right.$, Figure $\left.3 \mathrm{~B}\right)$. The mean absolute errors (MAE) for both correlations are comparable (13 and $17 \mathrm{~kJ} / \mathrm{mol}$, respectively) and are of the
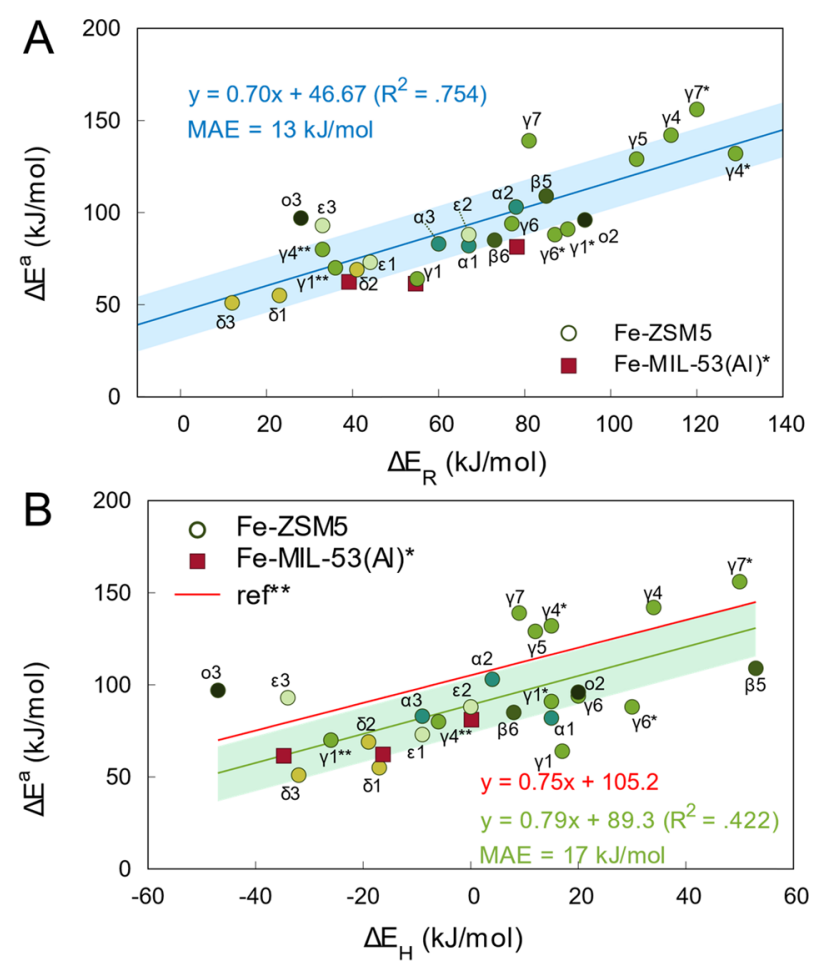

Figure 3. Relationships between the computed kinetic $\left(\Delta E^{a}\right)$ and thermodynamic ((A) $\Delta E_{\mathrm{R}}$ and (B) $\Delta E_{\mathrm{H}}$ ) parameters of methane activation by binuclear Fe sites in ZSM-5 zeolite (circles) and FeMIL-53 MOF (squares). The trend lines shown in the graph provide liner fits for the data sets with $\pm 15 \mathrm{~kJ} / \mathrm{mol}$ shown with shaded areas. $(*$ ref $44, * *$ ref 13$)$.

same magnitude as the MAE of $11 \mathrm{~kJ} / \mathrm{mol}$ obtained for a much larger data set in ref 13 . Despite having similar slopes ( 0.75 and 0.79 , respectively) indicating the fundamental nature of the $\Delta E^{\mathrm{a}}-\Delta E_{\mathrm{H}}$ correlation, a $16 \mathrm{~kJ} / \mathrm{mol}$ shift in the intercept is observed compared to the scaling law reported in ref 13 . It is likely due to the different exchange-correlation functionals employed. Figure 3 indicates that the current correlation established for Fe-ZSM-5 zeolite also holds for a related FeMIL-53(Al) metal organic framework (MOF) catalytic system. $^{44}$

The results in Figure $3 \mathrm{~A}$ reveal substantial deviations of the computed activation barriers from the linear $\Delta E^{\mathrm{a}}-\Delta E_{\mathrm{R}}$ trend. For example, significantly (deviation $>15 \mathrm{~kJ} / \mathrm{mol}$ ) lower reactivity is predicted for $\mathbf{0} 3, \varepsilon 3, \gamma 7$, and $\gamma 7^{*}$ sites, while the $\gamma 1, \gamma 6^{*}$, and $\gamma 1^{*}$ sites are predicted to be substantially more reactive than could be deduced from the idealized $\Delta E^{\mathrm{a}}-\Delta E_{\mathrm{R}}$ scaling relationship. The deviations from the $\Delta E^{\mathrm{a}}-\Delta E_{\mathrm{H}}$ correlation are much more substantial. Such direct relationships between the thermodynamic and kinetic parameters generally hold for reactive systems characterized by similar shapes of the potential energy surfaces (PES), i.e., for the systems with well-defined reactive centers, the intrinsic chemistry of which determines the chemical behavior. The secondary interactions in a multisite and/or confined environment of the reactive complexes in zeolite pores can significantly impact the involved PES giving rise to notable deviations from the energy scaling relationships.

In the current case we note that effects such as secondary hydrogen bonding interactions at the di-Fe site and specific confinement of the activated complexes exerted by the zeolite pores are the main causes for the observed deviations from the 
Table 1. Semiquantitative Analysis of the Main Secondary Interactions Due to Confinement, Flexibility and Multifunctionality of the Active Site on Methane Activation and the Associated Deviations (in $\mathrm{kJ} / \mathrm{mol}$ ) from the Fundamental Linear Energy Relationships $^{a}$

\begin{tabular}{|c|c|c|c|c|c|c|c|c|}
\hline \multirow{2}{*}{ Location } & \multicolumn{2}{|c|}{ Confinement $^{[\mathrm{a}]}$} & \multicolumn{4}{|c|}{ Flexibility $^{[b]}$ and Multifunctionality ${ }^{[c]}$} & \multicolumn{2}{|c|}{$\begin{array}{l}\Delta E^{\mathrm{a}} \text { deviation }(\mathrm{kJ} / \mathrm{mol}) \\
\text { from }\end{array}$} \\
\hline & $\mathrm{n}_{\mathrm{A}, T S}$ & $\mathrm{~d}_{\mathrm{C}-\mathrm{H}, F S}$ & $\begin{array}{c}\text { H-bond break. } \\
\text { @ TS }\end{array}$ & $\begin{array}{l}\text { H-bond form. } \\
\text { (a) 'site- } \mathrm{H}+{ }^{\circ} \mathrm{CH}_{3} \text { ' }\end{array}$ & $\begin{array}{l}\text { H-bond form. } \\
\text { @ 'site- } H \text { ' }\end{array}$ & OH-cooperativity & $\Delta E^{\mathrm{a}}-\Delta E_{\mathrm{R}}$ & $\Delta E^{\mathrm{a}}-\Delta E_{\mathrm{H}}$ \\
\hline$\alpha 1$ & 2 & 2.24 & - & - & - & - & -11 & -19 \\
\hline$\alpha 2$ & 7 & 2.30 & - & - & - & - & 1 & 10 \\
\hline$\alpha 3$ & 3 & 2.06 & - & - & - & - & -6 & 1 \\
\hline$\beta 5$ & 5 & 2.15 & - & - & + & - & 3 & -22 \\
\hline$\beta 6$ & 5 & 2.08 & - & - & + & + & -13 & -10 \\
\hline$\gamma 1$ & 4 & 2.08 & - & - & - & + & -21 & -38 \\
\hline$\gamma 1^{*}$ & 13 & 1.64 & - & - & - & - & -19 & -10 \\
\hline$\gamma 1 * *$ & 5 & 1.96 & - & + & - & + & -2 & 1 \\
\hline$\gamma 4$ & 12 & 2.48 & - & - & - & - & 16 & 26 \\
\hline$\gamma 4 *$ & 8 & 1.77 & - & - & + & - & -5 & 31 \\
\hline$\gamma 4 * *$ & 6 & 1.97 & - & + & - & + & 10 & -5 \\
\hline$\gamma 5$ & 11 & 1.81 & - & - & + & - & 8 & 31 \\
\hline$\gamma 6$ & 12 & 1.89 & - & - & - & + & -7 & -11 \\
\hline$\gamma 6^{*}$ & 6 & 2.10 & - & - & - & + & -19 & -25 \\
\hline$\gamma 7$ & 12 & 2.27 & - & - & - & - & 36 & 43 \\
\hline$\gamma 7^{*}$ & 9 & 2.52 & - & - & - & - & 25 & 28 \\
\hline$\delta 1$ & 3 & 2.04 & - & - & - & + & -8 & -21 \\
\hline$\delta 2$ & 1 & 1.86 & - & - & - & - & -11 & -5 \\
\hline$\delta 3$ & 2 & 2.07 & - & - & - & - & -4 & -13 \\
\hline$\varepsilon 1$ & 5 & 2.39 & - & - & - & - & -4 & -9 \\
\hline$\varepsilon 2$ & 1 & 2.17 & - & - & - & - & -6 & -2 \\
\hline$\varepsilon 3$ & 5 & 2.71 & + & - & - & - & 24 & 31 \\
\hline 02 & 4 & 1.85 & - & - & + & + & -16 & -9 \\
\hline 03 & 3 & 2.09 & + & - & - & - & 30 & 44 \\
\hline $\begin{array}{c}\Delta E^{\mathrm{a}}-\Delta E_{\mathrm{R}} \\
\text { regression coefs } \\
\text { [d] } \\
\Delta E^{\mathrm{a}}-\Delta E_{\mathrm{H}} \\
\text { regression coefs } \\
\text { [d] }\end{array}$ & $1.61^{(*)}$ & $23.5^{(*)}$ & $22.9^{(*)}$ & $19.2^{(*)}$ & - & $-11.5^{(*)}$ & & \\
\hline
\end{tabular}

${ }^{a_{T}}$ The occurrence of the particular effect for the given reaction is indicated with a + sign. The direction of the deviation is indicated with the color code defined in Figure 2 with the red and blue colour highlighting, respectively, the increase and decrease of the $\Delta E^{a}$ compared to the trend given by the the linear scaling law. ${ }^{[a]}$ Confinement effects in TS correlate with the number of atoms $n_{\mathrm{A}, \mathrm{TS}}$ from the zeolite lattice within a $3.5 \AA$ sphere around the $\mathrm{C}$ center, while the confinement effects in the $\mathrm{FS}$ are better described by the distance $d_{\mathrm{C}-\mathrm{H}, \mathrm{FS}}$ (in $\AA$ ) between the $\mathrm{C}$ atom in ${ }^{\circ} \mathrm{CH}_{3}$ and newly formed $\mathrm{H}$ species. ${ }^{[b]}$ The effects of active site flexibility on the reaction mechanism in the current system are associated with the rearrangement of the $\mathrm{H}$-bond networks in the course of the reaction such as the $\mathrm{H}$-bond cleavage in the TS and $\mathrm{H}$-bond stabilization of the FS and site-H, manifesting themselves in, respectively, $\Delta E^{\mathrm{a}}-\Delta E_{\mathrm{R}}$ and $\Delta E^{\mathrm{a}}-\Delta E_{\mathrm{H}}$ correlations. ${ }^{[c]}$ The presence of different functional groups within the active center allow for an alternative $\mathrm{OH}$-cooperative mechanism of methane activation. ${ }^{[d] *}$ and $* *$ after parameter value designates $p<0.05$ and $p<0.01$ significance levels, respectively; strikethrough means that the corresponding parameter was not used in the multiple regression. $\mathrm{R}^{2}=0.70$ and adjusted $\mathrm{R}^{2}=0.62$ for $\Delta E^{\mathrm{a}}-\Delta E_{\mathrm{R}}$ regression, and $\mathrm{R}^{2}=0.60$ and adjusted $\mathrm{R}^{2}=0.55$ for $\Delta E^{\mathrm{a}}-\Delta E_{\mathrm{H}}$ one.

idealized energy trends. The stabilizing and destabilizing effects associated with these secondary interactions are summarized schematically in Figure 2. The stabilization of the TS and the destabilization of the product state employed in a particular correlation (i.e., site- $\mathrm{H}+{ }^{\bullet} \mathrm{CH}_{3}$ for $\Delta E^{\mathrm{a}}-\Delta E_{\mathrm{R}}$ and site- $\mathrm{H}$ for $\left.\Delta E^{\mathrm{a}}-\Delta E_{\mathrm{H}}\right)$ results in a favorable deviation from the linear scaling (a lower activation energy), whereas the destabilization of the TS and the stabilization of the product state has an opposite effect on the activation energy. It should be noted, that in terms of $\Delta E^{\mathrm{a}}-\Delta E_{\mathrm{R} / \mathrm{H}}$ correlations $\Delta E^{\mathrm{a}}$ increasing is equivalent to $\Delta E_{\mathrm{R} / \mathrm{H}}$ decreasing and vice versa, therefore further analysis was performed from the $\Delta E^{\text {a }}$ deviation point of view only. Our calculations point to two main factors, namely the confinement and $\mathrm{H}$-bonding rearrangement, causing the deviations of the predicted reactivity from the idealized scaling law reflecting the intrinsic reactivity of the localized catalytic site. The results of a semiquantitative analysis of the nature and impact of these factors and their more specific contributions are summarized in Table 1 . Below we will discuss in detail the impact of each of the factors on the predicted reactivity of zeolite-stabilized di-Fe complexes in homolytic $\mathrm{C}-\mathrm{H}$ activation of methane.

Confinement Effects. The confinement effects due to the constraints of the zeolite micropores can manifest in destabilization of (i) the TS and (ii) the product site- $\mathrm{H}+$ ${ }^{-} \mathrm{CH}_{3}$ states. The former scenario is realized when the zeolite walls exert geometrical constraints to $\mathrm{CH}_{4}$ approaching the reactive $\mathrm{O}$-center, resulting in the effective destabilization of 
the transition state. To establish a (semi)quantitative descriptor for such a confinement effect, we consider the number of atoms excluding the $\mathrm{H}$ atoms of methane $\left(n_{\mathrm{A}, \mathrm{TS}}\right.$, Table 1) from the zeolite lattice and reaction center within 3.5 $\AA$ from the $\mathrm{C}$ atom of $\mathrm{CH}_{4}$ fragment. We note that increasing the number of atoms close to the reactive moiety in the TS increases $\Delta E^{\mathrm{a}}$ deviation by 1.6 and $2.8 \mathrm{~kJ} / \mathrm{mol}$ for $\Delta E^{\mathrm{a}}-\Delta E_{\mathrm{R}}$ and $\Delta E^{\mathrm{a}}-\Delta E_{\mathrm{H}}$ correlations, respectively, which reflects the decreased accessibility of the active site by $\mathrm{CH}_{4}$.

An illustrative example of this destabilization mechanism is the TS for the $\gamma 6$ site and TS- $\gamma 4 *$ shown in Figure 4A and C.

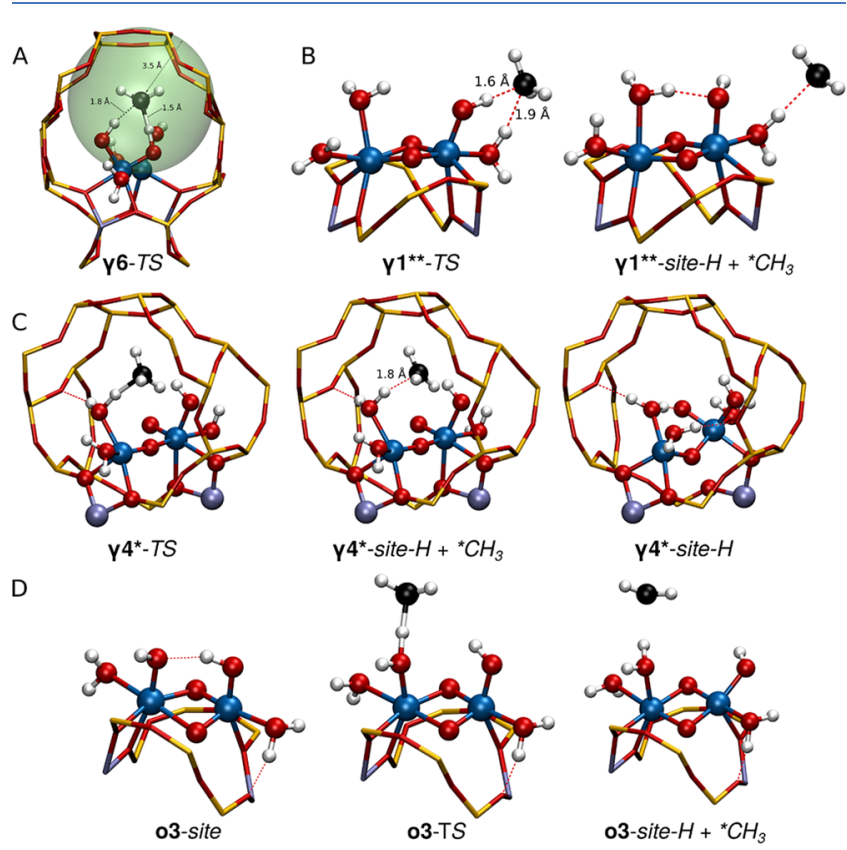

Figure 4. Representative cases for all identified deviation mechanisms. (A) Local structure of the TS of methane $\mathrm{C}-\mathrm{H}$ bond activation at the location of $\gamma 6$. The green sphere indicates totally 12 atoms from framework and reaction center distributed in the radius of $3.5 \AA$ of the $\mathrm{C}$ atom indicating that the TS is confined. H-bonding interaction of $T S$ with a close-by $\mathrm{OH}$ group is also highlighted. (B) Steps of reaction $\gamma 1^{* *}$. In the transition state there are two $\mathrm{OH}$ groups close to the forming $\mathrm{CH}_{3}$ radical. There is an additional $\mathrm{H}$ bond present compared to TS stabilizing this intermediate in site- $\mathrm{H}+{ }^{\bullet} \mathrm{CH}_{3}$. C) Steps of reaction $\gamma 4^{*}$ with a confined TS and a confined site- $\mathrm{H}+$ ${ }^{-} \mathrm{CH}_{3}$ where the $\mathrm{H}-\mathrm{C}$ distance is $1.8 \AA$. In the reduced site- $\mathrm{H}$ the $\mathrm{H}$ subtracted from the methane rotates and forms an additional $\mathrm{H}$ bond with the terminal $\mathrm{O}$ of the other $\mathrm{Fe}$ ion thus stabilizing site-H. D) Steps of reaction o3. In site there is a H-bond between the two $\mathrm{OH}$ ligands of Fe which breaks in the transition state.

Both structures have 12 neighboring atoms within $3.5 \AA$ from the reactive $\mathrm{C}$ atom. In these geometries, the rigid zeolite framework prevents $\mathrm{CH}_{4}$ from approaching the active O-site from the optimal angle thus increasing the activation barrier. We identify that TS confinement is the sole factor giving rise to the pronounced deviations from the linear scaling relationships for the sites $\gamma 4, \gamma 7$, and $\gamma 7^{*}$, for which the $\mathrm{C}-\mathrm{H}$ activation barriers are 16 to $43 \mathrm{~kJ} / \mathrm{mol}$ higher than those predicted based on the $\Delta E^{\mathrm{a}}-\Delta E_{\mathrm{R}}$ and $\Delta E^{\mathrm{a}}-\Delta E_{\mathrm{H}}$ correlations.

However, the number of atoms in the vicinity of the active site cannot be viewed as a universal indicator for the unfavorable impact of zeolite confinement. Previous theoretical studies indicate a pronounced reactivity dependence for homolytic $\mathrm{C}-\mathrm{H}$ cleavage on the angle at which the reactant approaches the reactive center. ${ }^{45}$ Depending on the $\mathrm{Fe}-\mathrm{O}-$ $\mathrm{HCH}_{3}$ angle, the reaction can proceed either via the $\sigma$-channel and involve the interaction with the $\mathrm{Fe}\left(\mathrm{d}_{\mathrm{z} 2}\right)+\mathrm{O}\left(\mathrm{p}_{z}\right)$ orbital or via the commonly much less favorable $\pi$-channel, involving the interaction with the $\mathrm{Fe}\left(\mathrm{d}_{x z / \mathrm{yz}}\right)-\mathrm{O}\left(\mathrm{p}_{\mathrm{x} / \mathrm{y}}\right)$ orbital (additional explanation can be found in ref 46 and Figure S3). Restricted accessibility of the reactive oxygen center due to confinement can flatten the associated PES and make the reactivity along the two channels comparable. However, an optimal angle of attack providing the favorable orbital overlap may still potentially be established despite the crowdedness at the active center. The only active site showing such a favorable geometry is $\gamma \mathbf{1}^{*}$ (Figure S4), for which, despite having the highest $n_{\mathrm{A}, \mathrm{TS}}$ among the structures considered here, the activation energy shows a favorable deviation in both linear correlations.

The geometrical constraints on the TS also affect the energetics of the associated site- $\mathrm{H}+{ }^{\bullet} \mathrm{CH}_{3}$ product state. The equilibrium distance between the ${ }^{\circ} \mathrm{CH}_{3}$ radical and the $\mathrm{HO}$ moiety of the partially reduced site, $d_{\mathrm{C}-\mathrm{H}, \mathrm{FS}}$, is ca. $2.0 \AA$ in the absence of the geometrical constraints due to zeolite pores. The increased confinement limits the mobility of ${ }^{\bullet} \mathrm{CH}_{3}$ resulting in shorter ${ }^{\circ} \mathrm{CH}_{3} \cdots \mathrm{HO}$ distances and, accordingly, increased energy of the site- $\mathrm{H}+{ }^{\bullet} \mathrm{CH}_{3}$ state by $23.6 \mathrm{~kJ} / \mathrm{mol}$ per $1 \AA$ decreasing ${ }^{\bullet} \mathrm{CH}_{3} \cdots \mathrm{HO}$ distance (or $2.36 \mathrm{~kJ} / \mathrm{mol}$ per 0.1 $\AA$ ). Such situations are encountered in, for example, sites $\gamma 4^{*}$ (Figure 4C), $\gamma 1^{*}$ and $\gamma 5$ (Figure S5). Importantly, the reactivity deviations induced by the confinement-induced transition (TS) and product (FS) states destabilization are very different. The TS confinement gives rise to an unfavorable reactivity shift to the red zone, while the FS confinement, on contrary, places the reactive system into the blue zone (Figure 1B). The mutual cancelation of these two effects is the main reason for the higher linearity of the $\Delta E^{\mathrm{a}}-\Delta E_{\mathrm{R}}$ correlation. The $\Delta E^{\mathrm{a}}-\Delta E_{\mathrm{H}}$ correlation does not account for the FS confinement and associated energy effects.

Confinement effects manifest themselves in the enthalpic and entropic components of the reaction and activation free energies. ${ }^{47,48}$ Here we considered only the enthalpic effects associated with the changes in the PES shape and therefore reflecting changes in the intrinsic reactivity of the active site. Previous studies proposed approaches for the quantification of the confinement effects ${ }^{49,50}$ via the analysis of the reaction energetics computed using full periodic and cluster zeolite models. Our analysis points to semiquantitative geometric descriptors for the confinement-induced deviations from the 1D linear scaling relations. However, other effects, especially those related to the flexibility and conformational freedom of the reactive ensemble are difficult to predict whether they occur during the transformations, and it is even more difficult to assign an adequate numerical descriptor. To identify representative quantitative descriptors for such effects a detailed analysis of much larger data sets than those considered in this study has to be carried out.

$\mathrm{H}$-Bonding Rearrangement. The $\mathrm{H}$-bonding rearrangement in the course of the reaction is another important factor affecting the shapes of the PES and giving rise to deviations from the linear energy scaling relationships. Calculations reveal that most reaction channels over the active sites featuring multiple $\mathrm{OH}$ ligands and coordinated $\mathrm{H}_{2} \mathrm{O}$ involve the concomitant cleavage and formation of hydrogen bonds, which, depending on the specific situation, may give rise to both favorable and unfavorable deviations from the predicted 
reactivity. We have distinguished 4 scenarios of such $\mathrm{H}$ bonding rearrangements: (i) $\mathrm{H}$-bond cleavage at TS, (ii) $\mathrm{H}$ bond stabilization of the FS, (iii) $\mathrm{H}$-bond stabilization of the partially reduced state site- $\mathrm{H}$, and (iv) $\mathrm{OH} / \mathrm{H}_{2} \mathrm{O}$ cooperative $\mathrm{C}-\mathrm{H}$ cleavage.

Terminal hydroxyl ligands on the binuclear Fe complexes have been identified as the potential sites for homolytic $\mathrm{C}-\mathrm{H}$ activation. ${ }^{39}$ The formation of $\mathrm{H}$-bonds between these ligands and neighboring proton-donating/accepting sites provide an additional stabilization to the complex (see, e.g., Figure 4D). However, during the $\mathrm{C}-\mathrm{H}$ activation process, these $\mathrm{H}$-bonds need to be broken at the TS to allow for the favorable orbital overlap between the reacting species. The associated energy losses are not recovered in the product states. The loss of such favorable $\mathrm{H}$-bond interaction during methane activation destabilizes both the TS and site- $\mathrm{H}+{ }^{\circ} \mathrm{CH}_{3}$ states. The destabilization is much more pronounced in the TS state as indicated by the consistent negative deviation (by 23 and 38 $\mathrm{kJ} / \mathrm{mol}$ into red zone for $\Delta E^{\mathrm{a}}-\Delta E_{\mathrm{R}}$ and $\Delta E^{\mathrm{a}}-\Delta E_{\mathrm{H}}$, respectively) of the predicted reactivity for the sites featuring this effect ( $\boldsymbol{\varepsilon} 3$ and $\mathbf{0 3}$, Figure 4D). The corresponding regression coefficients $(23$ and $38 \mathrm{~kJ} / \mathrm{mol}$ ) are close to the energies of moderate and strong $\mathrm{H}$-bonds, that additionally justifies the obtained model.

The partial reduction of the binuclear cationic complex with $\mathrm{CH}_{4}$ can be accompanied by the configurational changes in the active site resulting in additional $\mathrm{H}$-bonds between the terminal $\mathrm{OH}, \mathrm{O}$, or $\mathrm{H}_{2} \mathrm{O}$ ligands. This stabilizes the site$\mathrm{H}\left(+^{\bullet} \mathrm{CH}_{3}\right)$ and shifts the reaction energy $\left(\Delta E^{\mathrm{R}}\right)$ to the lefthand side of the BEP relationship (Figure 2), resulting in 19.2 $\mathrm{kJ} / \mathrm{mol} \Delta E^{\mathrm{a}}$ deviation. Representative examples are the reactions at sites $\gamma \mathbf{1}^{* *}$ and $\boldsymbol{\gamma} \mathbf{4}^{* *}$ (Table 1$)$. The optimized TS and site- $\mathrm{H}+{ }^{\bullet} \mathrm{CH}_{3}$ structures for $\gamma \mathbf{1}^{* *}$ are shown in Figure $4 \mathrm{~B}$. The reactive $\mathrm{Fe}$ complex in this case bears two $\mu$-O ligands, three terminal $\mathrm{H}_{2} \mathrm{O}$ and an oxo ligand. These ligands do not form intermolecular hydrogen bonds either in the pristine state site or in the TS state. Upon the reduction, the geometry of the cluster changes: most notably, the distance between the $\mathrm{Fe}$ centers decreases with the concomitant change in the relative configuration of the ligands so that an $\mathrm{H}$-bonding can now be established between the newly formed $\mathrm{Fe}_{1}-\mathrm{OH}$ and $\mathrm{Fe}_{2}-\mathrm{OH}_{2}$ moieties.

The geometry of the reduced site can further alter after the removal of the $\mathrm{CH}_{3}$ radical (Table 1 column 6). This situation is illustrated in Figure 4C with the optimized structures of TS, site- $\mathrm{H}+{ }^{\bullet} \mathrm{CH}_{3}$ and site- $\mathrm{H}$ for reaction $\gamma 4^{*}$. The relaxation of the reduced di-Fe complex after the removal of steric constraints imposed by the weakly bound ${ }^{\bullet} \mathrm{CH}_{3}$ species stabilizes the site$\mathrm{H}$ reference state with additional $\mathrm{H}$-bonds. As a result of these secondary interactions, lower values of $\mathrm{H}$-affinity are computed for such sites, which, however, is not related to the increased reactivity of the hydrogen-accepting moiety. We expected to observe this effect in the $\Delta E^{\mathrm{a}}-\Delta E_{\mathrm{H}}$ correlation only, where a deviation toward a red zone is commonly observed. However, both regression models do not contain the corresponding term. We suppose the reason is that this H-bonding stabilization effect was not observed independently without contributions from other (de)stabilization effects for the different active site locations considered in this study.

Multifunctionality. Multifunctionality of the active site can also cause deviations from the linear scaling relationship. Our DFT calculations suggest that the intrinsic reactivity of the $\mathrm{Fe}-\mathrm{O}$ moieties toward the homolytic $\mathrm{C}-\mathrm{H}$ dissociation can be influenced by the presence of vicinal $\mathrm{OH}$ and $\mathrm{H}_{2} \mathrm{O}$. Such a multifunctional environment allows for a more efficient stabilization of the $\mathrm{CH}_{3}$ radical formed in the TS through secondary interactions with the $\mathrm{OH} / \mathrm{H}_{2} \mathrm{O}$. This, in turn, leads to a decrease of the activation barrier for the reaction as compared to a hypothetical single-site mechanism (Table 1 column 7). Figures $4 \mathrm{~A}$ and $\mathrm{B}$ show the representative optimized structures of such cooperative TSs for $\gamma 6$ and $\gamma 1^{* *}$. The interatomic distance for the "activated" $\mathrm{C}-\mathrm{H}$ bond $\left(\mathrm{OH} \cdots{ }^{\bullet} \mathrm{CH}_{3}, 1.5-1.6 \AA\right)$ is comparable to that formed as a secondary interaction between the $\mathrm{CH}_{3}$ radical and a vicinal OH moiety (1.8-1.9 ̊). A substantial TS stabilization due to such a cooperative reaction mechanism is observed for the structures with secondary $\mathrm{r}\left(\mathrm{OH} \cdots{ }^{\bullet} \mathrm{CH}_{3}\right)<2.7 \AA$. The examples where this effect solely responsible for the deviations from the linear energy relations are the reactions $\gamma 1, \gamma 6^{*}$ and $\boldsymbol{\delta} \mathbf{1}$ (Figure S6). This cooperative methane activation mechanism has an impact on both linear relationships resulting in computed activation barriers 12 and $19 \mathrm{~kJ} / \mathrm{mol}$ lower than the ones predicted from the $\Delta E^{\mathrm{a}}-\Delta E_{\mathrm{R}}$ and $\Delta E^{\mathrm{a}}-\Delta E_{\mathrm{H}}$ linear fits, respectively.

Multiple regressions models obtained for $\Delta E^{\text {a }}$ deviation from the $\Delta E^{\mathrm{a}}-\Delta E_{\mathrm{R}}$ and $\Delta E^{\mathrm{a}}-\Delta E_{\mathrm{H}}$ correlations are characterized by 0.70 and $0.60 \mathrm{R}^{2}$ values, respectively. These values are not high enough to claim a nearly ideal explanation of the analyzed phenomenon. However, we argue that the correlation described provide crucial qualitative confirmation of our hypotheses. Importantly, all these effects manifest themselves simultaneously. For example, the confinement of both TS and site- $\mathrm{H}+{ }^{\circ} \mathrm{CH}_{3}$, or the TS confinement and $\mathrm{OH}$-cooperative methane activation mechanism can be encountered in the same system, and effectively cancel out or amplify the individual energy (de)stabilization effect. This can either give rise to the increased deviation from the trend line or, on contrary, to an apparent improvement of the linearity in the energy relation. For example, the TS and site- $\mathrm{H}+{ }^{\circ} \mathrm{CH}_{3}$ confinement destabilize both the transition and the final state for $\gamma 4^{*}$ (Figure 4C) and $\gamma \mathbf{5}$ (Figure S5), which reactivity perfectly follow the $\Delta E^{\mathrm{a}}-\Delta E_{\mathrm{R}}$ scaling trend. However, site-H for these configurations is additionally stabilized by $\mathrm{H}$-bonding, which causes substantial deviations in the $\Delta E^{\mathrm{a}}-\Delta E_{\mathrm{H}}$ relationship. For the sites $\gamma 1^{* *}$ and $\gamma 4^{* *}$ the opposing effects of $\mathrm{OH}$ cooperativity group and $\mathrm{H}$-bond rearrangements during methane activation cancel each other out as is evident from the minimal deviation of the corresponding computed and expected energetics (Table 1).

\section{CONCLUSIONS}

Fundamental reactivity theory implies that there is a relationship between thermodynamics and kinetics when the intrinsic chemistry of the active site is in charge of the activity. However, this study identifies that besides the intrinsic reactivity of the active site, there is a number of secondary effects present in microporous catalytic materials that can outweigh the intrinsic chemistry and break the scaling relationships. (i) The confinement of porous frameworks does have an effect on the reaction barrier. Confinement of the transition state limits the geometry flexibility, and thus increases the reaction barrier. (ii) H-bond breaking or forming leads to the destabilization or stabilization of the reaction intermediates, moving the points in Figure 2 away from the scaling relationships. (iii) Direct stabilization of the transition state was observed when the interaction of a close-by $\mathrm{OH}$ 
group and the $\mathrm{CH}_{3}$ radical was realized. This interaction lowers the energy of the transition state and breaks the scaling relations, which is proposed to be a practical strategy for catalyst design toward methane activation.

\section{ASSOCIATED CONTENT}

\section{(5) Supporting Information}

The Supporting Information is available free of charge on the ACS Publications website at DOI: 10.1021/acscatal.9b01914.

Local geometry of the $\left[\left(\mathrm{H}_{2} \mathrm{O}\right)_{2}-\mathrm{Fe}(\mathrm{III})-(\mu \mathrm{O})_{2}-\mathrm{Fe}\right.$ (III) $\left.-\left(\mathrm{H}_{2} \mathrm{O}\right)_{2}\right]^{2+}$ cluster, all locations for stabilization of the active site, a detailed explanation about the $\pi$ and $\sigma$ channel of $\mathrm{C}-\mathrm{H}$ bond activation of methane, local geometries of the selected transition states, BEP relationship, and free energy calculations (PDF)

\section{AUTHOR INFORMATION}

\section{Corresponding Author}

*E-mail: e.a.pidko@tudelft.nl.

\section{ORCID $\odot$}

Ivan Yu. Chernyshov: 0000-0002-4452-2025

Guanna Li: 0000-0003-3031-8119

Jorge Gascon: 0000-0001-7558-7123

Evgeny A. Pidko: 0000-0001-9242-9901

\section{Present Address}

"Inorganic Systems Engineering Group, Department of Chemical Engineering, Delft University of Technology, Van der Maasweg 9, 2629 HZ Delft, The Netherlands.

\section{Notes}

The authors declare no competing financial interest.

\section{ACKNOWLEDGMENTS}

The Dutch Science Foundation (NWO) is gratefully acknowledged for financial support through the VIDI personal grant MetMOFCat. G.L. acknowledges financial support from NWO for her personal VENI grant (016.Veni.172.034). E.K. and I.Y.C. acknowledge partial support from the Ministry of Education and Science of the Russian Federation (Project $11.1706 .2017 / 4.6)$. I.Y.C. is deeply grateful to the first reviewer for the opportunity to become one of the coauthors of this work. NWO is acknowledged for providing access to SurfSARA supercomputer resources.

\section{REFERENCES}

(1) Greeley, J. Theoretical Heterogeneous Catalysis: Scaling Relationships and Computational Catalyst Design. Annu. Rev. Chem. Biomol. Eng. 2016, 7, 605-635.

(2) Golodets, G. I. Relationship between the Catalytic and Thermodynamic Properties of Substances. In Heterogeneous Catalytic Reactions Involving Molecular Oxygen; Golodets, G. I., Ed.; Elsevier: 1983; Vol. 15, pp 151-178.

(3) Calle-Vallejo, F.; Loffreda, D.; Koper, M. T. M.; Sautet, P. Introducing structural sensitivity into adsorption-energy scaling relations by means of coordination numbers. Nat. Chem. 2015, 7, 403.

(4) Xin, H.; Vojvodic, A.; Voss, J.; Nørskov, J. K.; Abild-Pedersen, F. Effects of $d$-band shape on the surface reactivity of transition-metal alloys. Phys. Rev. B: Condens. Matter Mater. Phys. 2014, 89, 115114.

(5) Duyar, M. S.; Tsai, C.; Snider, J. L.; Singh, J. A.; Gallo, A.; Yoo, J. S.; Medford, A. J.; Abild-Pedersen, F.; Studt, F.; Kibsgaard, J.; Bent, S. F.; Nørskov, J. K.; Jaramillo, T. F. A Highly Active Molybdenum Phosphide Catalyst for Methanol Synthesis from $\mathrm{CO}$ and $\mathrm{CO}_{2}$. Angew. Chem., Int. Ed. 2018, 57, 15045-15050.
(6) Barteau, M. A. Linear free energy relationships for C1-oxygenate decomposition on transition metal surfaces. Catal. Lett. 1991, 8, 175183.

(7) Logadottir, A.; Rod, T. H.; Nørskov, J. K.; Hammer, B.; Dahl, S.; Jacobsen, C. J. H. The Brønsted-Evans-Polanyi Relation and the Volcano Plot for Ammonia Synthesis over Transition Metal Catalysts. J. Catal. 2001, 197, 229-231.

(8) Nørskov, J. K.; Bligaard, T.; Logadottir, A.; Bahn, S.; Hansen, L. B.; Bollinger, M.; Bengaard, H.; Hammer, B.; Sljivancanin, Z.; Mavrikakis, M.; Xu, Y.; Dahl, S.; Jacobsen, C. J. H. Universality in Heterogeneous Catalysis. J. Catal. 2002, 209, 275-278.

(9) Tran, K.; Ulissi, Z. W. Active learning across intermetallics to guide discovery of electrocatalysts for $\mathrm{CO} 2$ reduction and $\mathrm{H} 2$ evolution. Nat. Catal. 2018, 1, 696-703.

(10) Ulissi, Z. W.; Medford, A. J.; Bligaard, T.; Nørskov, J. K. To address surface reaction network complexity using scaling relations machine learning and DFT calculations. Nat. Commun. 2017, 8, 14621 .

(11) Grajciar, L.; Heard, C. J.; Bondarenko, A. A.; Polynski, M. V.; Meeprasert, J.; Pidko, E. A.; Nachtigall, P. Towards operando computational modeling in heterogeneous catalysis. Chem. Soc. Rev. 2018, 47, 8307-8348.

(12) Laursen, A. B.; Wexler, R. B.; Whitaker, M. J.; Izett, E. J.; Calvinho, K. U. D.; Hwang, S.; Rucker, R.; Wang, H.; Li, J.; Garfunkel, E.; Greenblatt, M.; Rappe, A. M.; Dismukes, G. C. Climbing the Volcano of Electrocatalytic Activity while Avoiding Catalyst Corrosion: $\mathrm{Ni}_{3} \mathrm{P}$, a Hydrogen Evolution Electrocatalyst Stable in Both Acid and Alkali. ACS Catal. 2018, 8, 4408-4419.

(13) Latimer, A. A.; Kulkarni, A. R.; Aljama, H.; Montoya, J. H.; Yoo, J. S.; Tsai, C.; Abild-Pedersen, F.; Studt, F.; Nørskov, J. K. Understanding trends in $\mathrm{C}-\mathrm{H}$ bond activation in heterogeneous catalysis. Nat. Mater. 2017, 16, 225.

(14) Olivos-Suarez, A. I.; Szécsényi, À.; Hensen, E. J. M.; RuizMartinez, J.; Pidko, E. A.; Gascon, J. Strategies for the Direct Catalytic Valorization of Methane Using Heterogeneous Catalysis: Challenges and Opportunities. ACS Catal. 2016, 6, 2965-2981.

(15) Bao, J.; Yang, G.; Yoneyama, Y.; Tsubaki, N. Significant Advances in C1 Catalysis: Highly Efficient Catalysts and Catalytic Reactions. ACS Catal. 2019, 9, 3026-3053.

(16) Dinh, K. T.; Sullivan, M. M.; Serna, P.; Meyer, R. J.; Dincă, M.; Román-Leshkov, Y. Viewpoint on the Partial Oxidation of Methane to Methanol Using $\mathrm{Cu}$ - and Fe-Exchanged Zeolites. ACS Catal. 2018, 8, 8306-8313.

(17) Li, G.; Vassilev, P.; Sanchez-Sanchez, M.; Lercher, J. A.; Hensen, E. J. M.; Pidko, E. A. Stability and reactivity of copper oxoclusters in ZSM-5 zeolite for selective methane oxidation to methanol. J. Catal. 2016, 338, 305-312.

(18) Mahyuddin, M. H.; Tanaka, T.; Shiota, Y.; Staykov, A.; Yoshizawa, K. Methane Partial Oxidation over $\left[\mathrm{Cu}_{2}(\mu-\mathrm{O})\right]^{2+}$ and $\left[\mathrm{Cu}_{3}(\mu-\mathrm{O})_{3}\right]^{2+}$ Active Species in Large-Pore Zeolites. ACS Catal. 2018, 8, 1500-1509.

(19) Rosen, A. S.; Notestein, J. M.; Snurr, R. Q. Structure-Activity Relationships that Identify Metal-Organic Framework Catalysts for Methane Activation. ACS Catal. 2019, 9, 3576-3587.

(20) Sun, G.; Zhao, Z.-J.; Mu, R.; Zha, S.; Li, L.; Chen, S.; Zang, K.; Luo, J.; Li, Z.; Purdy, S. C.; et al. Breaking the scaling relationship via thermally stable $\mathrm{Pt} / \mathrm{Cu}$ single atom alloys for catalytic dehydrogenation. Nat. Commun. 2018, 9, 4454-4454.

(21) Mehta, P.; Barboun, P.; Herrera, F. A.; Kim, J.; Rumbach, P.; Go, D. B.; Hicks, J. C.; Schneider, W. F. Overcoming ammonia synthesis scaling relations with plasma-enabled catalysis. Nat. Catal. 2018, 1, 269-275.

(22) Pegis, M. L.; Wise, C. F.; Koronkiewicz, B.; Mayer, J. M. Identifying and Breaking Scaling Relations in Molecular Catalysis of Electrochemical Reactions. J. Am. Chem. Soc. 2017, 139, 1100011003.

(23) Gani, T. Z. H.; Kulik, H. J. Understanding and Breaking Scaling Relations in Single-Site Catalysis: Methane to Methanol Conversion by $\mathrm{Fe}^{\mathrm{IV}}=\mathrm{O}$. ACS Catal. 2018, 8, 975-986. 
(24) Jiao, L.; Wang, Y.; Jiang, H.-L.; Xu, Q. Metal-Organic Frameworks as Platforms for Catalytic Applications. Adv. Mater. 2018, 30, 1703663.

(25) Gascon, J.; Corma, A.; Kapteijn, F.; Llabrés i Xamena, F. X. Metal Organic Framework Catalysis: Quo vadis? ACS Catal. 2014, 4, 361-378.

(26) John, M.; Alexopoulos, K.; Reyniers, M.-F.; Marin, G. B. FirstPrinciples Kinetic Study on the Effect of the Zeolite Framework on 1Butanol Dehydration. ACS Catal. 2016, 6, 4081-4094.

(27) Liu, C.; Tranca, I.; van Santen, R. A.; Hensen, E. J. M.; Pidko, E. A. Scaling Relations for Acidity and Reactivity of Zeolites. J. Phys. Chem. C 2017, 121, 23520-23530.

(28) Liang, T.; Chen, J.; Qin, Z.; Li, J.; Wang, P.; Wang, S.; Wang, G.; Dong, M.; Fan, W.; Wang, J. Conversion of Methanol to Olefins over H-ZSM-5 Zeolite: Reaction Pathway Is Related to the Framework Aluminum Siting. ACS Catal. 2016, 6, 7311-7325.

(29) Panov, G. I.; Uriarte, A. K.; Rodkin, M. A.; Sobolev, V. I. Generation of active oxygen species on solid surfaces. Opportunity for novel oxidation technologies over zeolites. Catal. Today 1998, 41, $365-385$.

(30) Hammond, C.; Forde, M. M.; Ab Rahim, M. H.; Thetford, A.; He, Q.; Jenkins, R. L.; Dimitratos, N.; Lopez-Sanchez, J. A.; Dummer, N. F.; Murphy, D. M.; Carley, A. F.; Taylor, S. H.; Willock, D. J.; Stangland, E. E.; Kang, J.; Hagen, H.; Kiely, C. J.; Hutchings, G. J. Direct Catalytic Conversion of Methane to Methanol in an Aqueous Medium by using Copper-Promoted Fe-ZSM-5. Angew. Chem., Int. Ed. 2012, 51, 5129-5133.

(31) Osadchii, D. Y.; Olivos-Suarez, A. I.; Szecsenyi, A.; Li, G. N.; Nasalevich, M. A.; Dugulan, J. A.; Crespo, P. S.; Hensen, E. J. M.; Veber, S. L.; Fedin, M. V.; Sankar, G.; Pidko, E. A.; Gascon, J. Isolated $\mathrm{Fe}$ Sites in Metal Organic Frameworks Catalyze the Direct Conversion of Methane to Methanol. ACS Catal. 2018, 8, 55425548.

(32) Kresse, G.; Furthmüller, J. Efficient iterative schemes for ab initio total-energy calculations using a plane-wave basis set. Phys. Rev. B: Condens. Matter Mater. Phys. 1996, 54, 11169-11186.

(33) Kresse, G.; Joubert, D. From ultrasoft pseudopotentials to the projector augmented-wave method. Phys. Rev. B: Condens. Matter Mater. Phys. 1999, 59, 1758-1775.

(34) Perdew, J. P.; Burke, K.; Ernzerhof, M. Generalized Gradient Approximation Made Simple. Phys. Rev. Lett. 1996, 77, 3865-3868.

(35) Blöchl, P. E. Projector augmented-wave method. Phys. Rev. B: Condens. Matter Mater. Phys. 1994, 50, 17953-17979.

(36) Vogiatzis, K. D.; Li, G.; Hensen, E. J. M.; Gagliardi, L.; Pidko, E. A. Electronic Structure of the $\left[\mathrm{Cu}_{3}(\mu-\mathrm{O})_{3}\right]^{2+}$ Cluster in Mordenite Zeolite and Its Effects on the Methane to Methanol Oxidation. J. Phys. Chem. C 2017, 121, 22295-22302.

(37) Grimme, S.; Ehrlich, S.; Goerigk, L. Effect of the damping function in dispersion corrected density functional theory. J. Comput. Chem. 2011, 32, 1456-1465.

(38) Henkelman, G.; Uberuaga, B. P.; Jónsson, H. A climbing image nudged elastic band method for finding saddle points and minimum energy paths. J. Chem. Phys. 2000, 113, 9901-9904.

(39) Szécsényi, Á.; Li, G.; Gascon, J.; Pidko, E. A. Mechanistic complexity of methane oxidation with $\mathrm{H} 2 \mathrm{O} 2$ by single-site $\mathrm{Fe} / \mathrm{ZSM}-5$ catalyst. ACS Catal. 2018, 8, 7961-7972.

(40) Belanzoni, P.; Bernasconi, L.; Baerends, E. J. $\mathrm{O}_{2}$ Activation in a Dinuclear Fe(II)/EDTA Complex: Spin Surface Crossing As a Route to Highly Reactive Fe(IV)oxo Species. J. Phys. Chem. A 2009, 113, 11926-11937.

(41) Li, G.; Pidko, E. A.; van Santen, R. A.; Li, C.; Hensen, E. J. M. Stability of Extraframework Iron-Containing Complexes in ZSM-5 Zeolite. J. Phys. Chem. C 2013, 117, 413-426.

(42) Woertink, J. S.; Smeets, P. J.; Groothaert, M. H.; Vance, M. A.; Sels, B. F.; Schoonheydt, R. A.; Solomon, E. I. A $\left[\mathrm{Cu}_{2} \mathrm{O}\right]^{2+}$ core in $\mathrm{Cu}-$ ZSM-5, the active site in the oxidation of methane to methanol. Proc. Natl. Acad. Sci. U. S. A. 2009, 106, 18908-18913.

(43) Ab Rahim, M. H.; Forde, M. M.; Jenkins, R. L.; Hammond, C.; He, Q.; Dimitratos, N.; Lopez-Sanchez, J. A.; Carley, A. F.; Taylor, S.
H.; Willock, D. J.; Murphy, D. M.; Kiely, C. J.; Hutchings, G. J. Oxidation of Methane to Methanol with Hydrogen Peroxide Using Supported Gold-Palladium Alloy Nanoparticles. Angew. Chem., Int. Ed. 2013, 52, 1280-1284.

(44) Szécsényi, Á.; Li, G.; Gascon, J.; Pidko, E. A. Unraveling reaction networks behind the catalytic oxidation of methane with $\mathrm{H}_{2} \mathrm{O}_{2}$ over a mixed-metal MIL-53(Al,Fe) MOF catalyst. Chem. Sci. 2018, 9, 6765-6773.

(45) Kazaryan, A.; Baerends, E. J. Ligand Field Effects and the High Spin-High Reactivity Correlation in the $\mathrm{H}$ Abstraction by NonHeme Iron(IV)-Oxo Complexes: A DFT Frontier Orbital Perspective. ACS Catal. 2015, 5, 1475-1488.

(46) Kazaryan, A.; Baerends, E. J. Ligand Field Effects and the High Spin-High Reactivity Correlation in the $\mathrm{H}$ Abstraction by NonHeme Iron(IV)-Oxo Complexes: A DFT Frontier Orbital Perspective. ACS Catal. 2015, 5, 1475-1488.

(47) Gounder, R.; Iglesia, E. The Roles of Entropy and Enthalpy in Stabilizing Ion-Pairs at Transition States in Zeolite Acid Catalysis. Acc. Chem. Res. 2012, 45, 229-238.

(48) Li, G.; Pidko, E. A. The Nature and Catalytic Function of Cation Sites in Zeolites: a Computational Perspective. ChemCatChem 2019, 11, 134-156.

(49) Göltl, F.; Michel, C.; Andrikopoulos, P. C.; Love, A. M.; Hafner, J.; Hermans, I.; Sautet, P. Computationally Exploring Confinement Effects in the Methane-to-Methanol Conversion Over Iron-Oxo Centers in Zeolites. ACS Catal. 2016, 6, 8404-8409.

(50) Mansoor, E.; Van der Mynsbrugge, J.; Head-Gordon, M.; Bell, A. T. Impact of Long-range Electrostatic and Dispersive Interactions on Theoretical Predictions of Adsorption and Catalysis in Zeolites. Catal. Today 2018, 312, 51-65. 